- There is a need to focus attention not only on prepared teeth but also the remaining teeth in the same arch when making impressions for indirect restorations.

- When using dual phase impressions, clinicians need to be careful when seating the loaded impression tray so as to avoid subsequent inaccuracies in dental casts.

- On the whole clinicians are better at making impressions for study and opposing casts than working crown and bridge impressions.

\title{
The quality of dental casts used in crown and bridgework
}

\author{
N. Alhouri, ${ }^{1}$ J. F. McCord ${ }^{2}$ and P. W. Smith ${ }^{3}$
}

\begin{abstract}
Objective To assess the quality of dental casts used in crown and bridge construction.

Design Observational cross-sectional study of dental casts. Setting Commercial dental laboratories and a university dental hospital laboratory in the UK.

Materials and methods A sample $(n=150)$ of working and opposing casts used for crown and bridgework prescribed by general dental practices and a dental hospital were sampled from two commercial dental laboratories and an 'on-site' university dental hospital laboratory respectively. A simple '3 point' assessment scale of quality (good, fair and poor) was used to categorise the casts depending on the clarity of reproduction of soft and hard tissues. Results The quality of opposing casts used for articulation purposes was significantly better $(\mathrm{P}<0.001)$ than that of the working casts. In addition it was found that for working casts the quality in the preparation area(s) was significantly better $(p<0.001)$ than that in areas remote from preparation(s) in the same arch. In general, the quality of casts in the incisal or occlusal surfaces was better than the buccal and lingual surfaces.

Conclusions This study has demonstrated that variation exists in the quality of casts used in crown and bridgework, specifically those used in the construction of indirect restorations and also those used for articulation purposes. This study highlights the need for clinicians to exercise continued vigilance with crown and bridge impressions, and casts, particularly in areas away from the prepared teeth.
\end{abstract}

\section{INTRODUCTION}

The indirect technique for fabricating crowns and bridges allows the fabrication of a restoration away from the chairside, by substituting a gypsum cast for the actual tooth. If the restoration is to fit the mouth precisely, the cast on which it is

${ }^{1}$ Lecturer, Damascus University, Syria, ${ }^{2}$ Professor/ Hon. Consultant in Restorative Dentistry, ${ }^{3 *}$ Lecturer/ Hon. Consultant in Restorative Dentistry, Unit of Prosthodontics, University Dental Hospital of Manchester, Manchester

*Correspondence to: Philip Smith, Unit of Prosthodontics, University Dental Hospital of Manchester, Manchester M15 6FH

Email:psmith@man.ac.uk

\section{Refereed paper}

Received 10.06.03; Accepted 8.10.03

doi:10.1038/sj.bdj.4811621

๑) British Dental Journal 2004; 194: 261-264 made must closely replicate the prepared tooth or teeth and associated areas of soft tissue.

Previous studies have examined working casts and used these as an indicator of the accuracy of impressions. ${ }^{1}$ It has been suggested that good dental casts should satisfy the following requirements: ${ }^{2}$

- They must be bubble free, especially along the finish lines of the prepared teeth

- All aspects must be distortion free

- They must be capable of being trimmed to allow access to the margins.

The British Society for Restorative Dentistry ${ }^{3}$ has indicated that the purpose of the master impression is to "obtain an accurate, dimensionally stable, fully-supported impression of the prepared teeth and associated soft tissues'. A full arch impression should normally be recorded to facilitate the articulation of working casts and to provide sufficient information in respect of occlusal form, function and relationships.

Apart from the need to accurately record the tooth preparations and associated soft tissues, it is also important to recognise that impressions of the opposing arch are also critical to the success of crown and bridgework. An accurate alginate impression will usually suffice for this, assuming that it is handled correctly and cast within the requisite time using appropriate gypsum based products.

Although the need for accuracy in crown and bridgework is generally appreciated by clinicians, studies have shown that a significant number of the impressions, from which the casts used for indirect restorations are made, demonstrate defects which might influence the quality of crowns or bridges. ${ }^{1,4,5}$

This study sought to determine the quality of dental casts used in crown and bridgework by sampling casts from commercial and dental hospital laboratories.

\section{MATERIAL AND METHODS}

A sample of 150 sets of working and corresponding opposing casts was drawn randomly from different laboratory sources; 100 sets from two different commercial dental laboratories (50 from each) that take dental work from throughout the United Kingdom, and 50 from a university dental hospital laboratory. They were analysed by a single observer. 


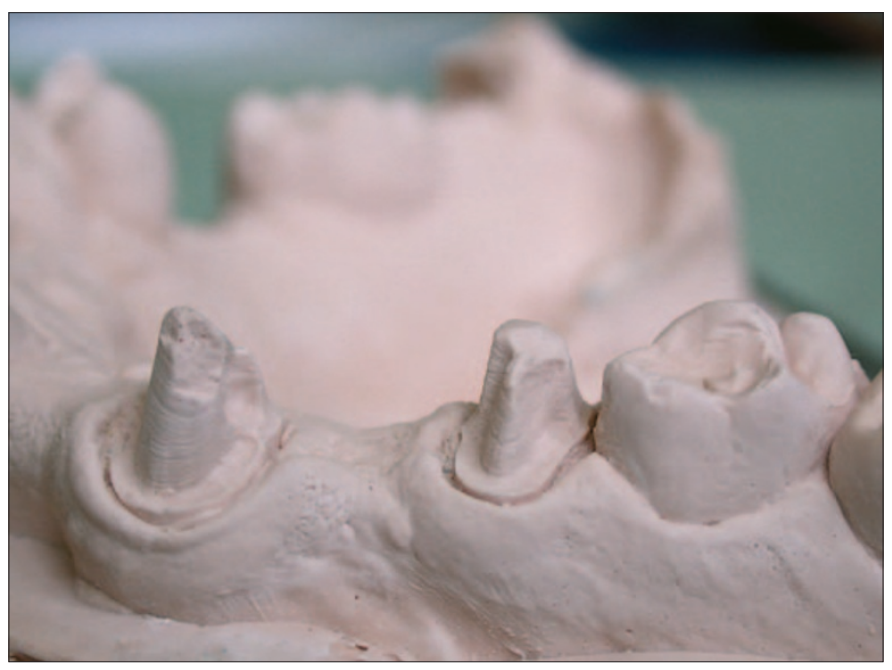

Fig. 1 Example of a dental cast showing 'good' reproduction of the details of soft and hard tissues

The teeth in each jaw were divided into six areas:

- right molars,

- left molars

- right premolars

- left premolars,

- right anteriors

- left anteriors.

Each of the above areas was further subdivided into three surfaces: buccal, occlusal, and lingual. The location of prepared teeth was also recorded for the working casts.

\section{Assessment of the quality of dental casts}

The surfaces were considered of good quality if the hard tissues (tooth) and soft tissues (gingival margins) were sound and free of any obvious distortion (Fig. 1). If the surfaces appeared intact but with minimal distortion, eg small bubbles or minor imperfections, they were considered to have fair quality (Fig. 2). Otherwise the surfaces were considered to have poor quality (Fig. 3).

\section{Cast quality score (COS)}

According to the cast quality score (CQS) used in this study:

CQS 1 represents good quality,

CQS 2 represents fair quality,

CQS 3 represents poor quality

Therefore, a lower CQS indicates better cast quality. The values of CQS of the working and opposing casts were calculated by the sum of the values of each surface divided by the number of the surfaces. Missing surfaces were not included in the analyses.

\section{RESULTS}

\section{Method error}

Measurement error was assessed by repeat measurements of 17 randomly selected dental casts with a one-week interval between. The agreement was assessed by means of the Kappa statistical value. The Kappa value in this study was 0.87 and the percentage agreement was 96\%. Any differences in the measurements were either between poor and fair categories, or between good and fair categories. No error measurement was found between good and poor categories. The CQS data were subjected to one-way ANOVA in order to test for differences in quality between working and opposing casts.

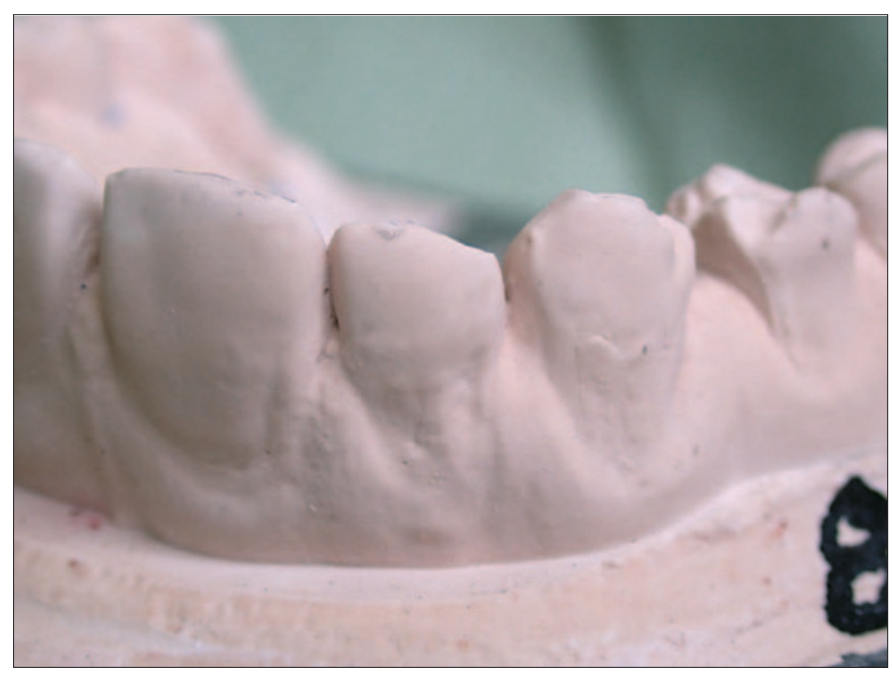

Fig. 2 Example of a dental cast showing 'fair' reproduction of the details of soft and hard tissues

The quality of working and opposing casts

- There was no significant difference $(\mathrm{P}=0.51)$ in the quality of working casts between those poured from dental student- or GDP- made impressions (Table 1).

- Opposing casts made from student impressions had significantly lower CQS $(\mathrm{P}=0.001)$ than those which originated from GDPs (Table 1).

- The preparation area(s) had significantly lower CQS $(\mathrm{P}<0.001)$ than the areas remote from preparation in the same arch (Table 2) for both groups.

It was also found, by examining the CQS of opposing casts that:

- The incisal and occlusal surfaces had better quality than the buccal and lingual surfaces in each area.

- The quality of casts decreases from anterior to posterior areas. The exception was the buccal anterior region which tended to demonstrate the poorest quality.

The overall quality of opposing casts was significantly better $(\mathrm{P}<0.001)$ than the working casts (Table 3$)$.

\section{DISCUSSION}

The high percentage agreement (96\%) between the first and second measurements found in this study demonstrates that the scoring system was likely to have been consistently applied throughout the study.

Table 1 Statistical analysis of the differences in quality between casts resulting from student and GDP made impressions

\begin{tabular}{lcc|l|c|c|c}
\hline Casts & \multicolumn{2}{c|}{$\mathrm{N}$} & Pvalue & \multicolumn{2}{c|}{ Mean (SD) } & Mean Diff \\
\hline & Student & GDP & & Student & GDP & \\
\hline Working & 50 & 100 & $P=0.51$ & $1.35(0.18)$ & $1.38(0.33)$ & -0.03 \\
Opposing & 50 & 100 & $P=0.001$ & $1.11(0.20)$ & $1.23(0.25)$ & -0.12
\end{tabular}

Table 2 Statistical analysis of the differences in casts' quality between preparation area(s) and the area(s) remote from preparation

\begin{tabular}{ll|l|c|l|l}
\hline Casts & $\mathrm{N}$ & Pvalue & \multicolumn{2}{|c|}{ Mean (SD) } & Mean Diff (SD) \\
\hline & & Preparation area & Remote areas & \\
\hline $\begin{array}{l}\text { Preparation and } \\
\text { remote areas }\end{array}$ & 150 & $\mathrm{P}<0.001$ & $1.13(0.28)$ & $1.50(0.37)$ & $-0.37(0.41)$ \\
\hline
\end{tabular}

Table 3 Statistical analysis of the differences in quality between working and opposing casts

\begin{tabular}{lc|c|c|c|c}
\hline Casts & $\mathrm{N}$ & Pvalue & \multicolumn{2}{|c|}{ Mean (SD) } & M ean Diff (SD) \\
\hline & & & Working casts & Opposing casts & \\
\hline $\begin{array}{l}\text { Working } \\
\text { and opposing }\end{array}$ & 150 & $P<0.001$ & $1.37(0.29)$ & $1.19(0.24)$ & $0.18(0.33)$ \\
\hline
\end{tabular}




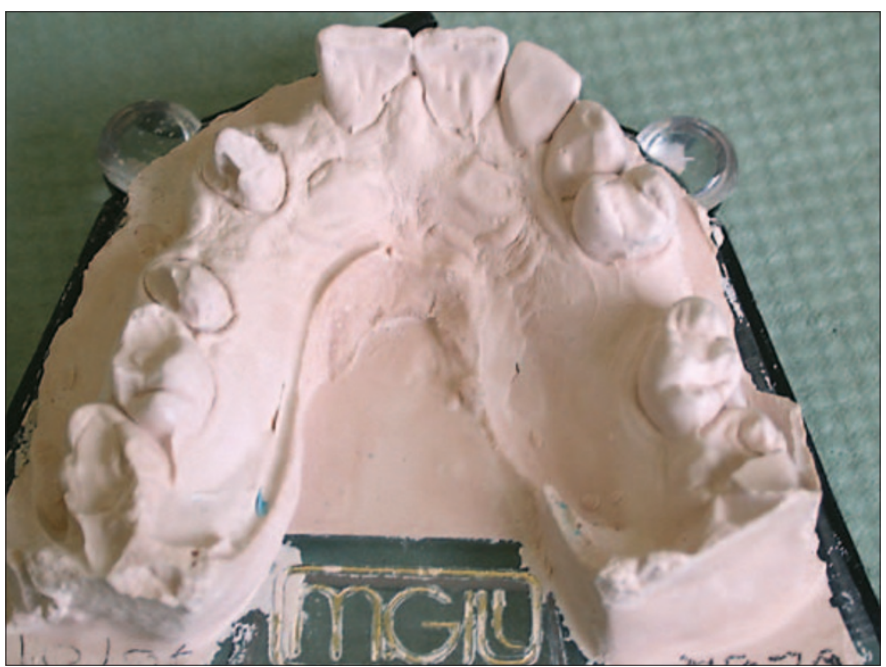

Fig. 3 Example of a dental cast showing 'poor' reproduction of the occlusal surfaces of last molars

Although this study did not seek to identify the type of impression material used, it was evident that most of the crown and bridge impressions had been made in silicone elastomer in stock impression trays. This reflects the findings of Randall et al. ${ }^{6}$ who demonstrated that the use of addition-cured silicones were found to predominate in undergraduate teaching; 71\% of schools taught and used a one-stage, full arch impression technique involving stock trays, and 57\% of schools a full-arch custom tray technique.

Although there was no significant difference $(\mathrm{P}=0.51)$ in the quality of the casts between student-made and GDP-made working casts, opposing casts made by students had significantly $(\mathrm{P}=0.001)$ lower $\mathrm{CQS}$ than those prescribed by GDPs. It is possible to speculate that this might have occurred because the students are likely to be under less time pressure than a busy GDP, and in addition undergraduate dental students have the support of an experienced clinical tutor who is able to offer direct assistance in difficult cases.

The quality of casts directly related to the preparation areas was significantly better $(\mathrm{P}<0.001)$ than that of the areas remote from the preparation in the same arch. Defects identified near the preparation area were seen mainly to be double layers resulting in a gap between the first and second phase of the impression material. This result appears to fit with the findings of Carrotte et $a l,{ }^{4}$ who found the majority of working impressions (42 out of 50) employed a dual impression technique, combining either heavy/light or putty/wash viscosities of material. Several of the impressions showed severe step defects between the two phases.

It was also noticed in the study reported here that the wash material tended to be used mostly in the preparation area, and not to record the detail of the occlusal surfaces of the other teeth in the same arch. This result indicates that greater care may need to be taken during the impression making stage, and also possibly subsequent laboratory casting procedures, in order to ensure that the required level of occlusal accuracy is achieved. ${ }^{7}$

Carrotte et al. ${ }^{4}$ also found defects in impressions of the preparations in 38 out of 50 cases. Apart from the impression of the preparation itself, 22 cases also showed defects in other parts of the impression. Similarly, Winstanley et al. ${ }^{5}$ found defects in the preparation area in the impression in $36.2 \%$ of the cases. The majority of defective preparations showed indefinite margins, and a number demonstrated blows, drags, and folds.

The quality of casts of the incisal or occlusal surfaces tended to be better than that of the buccal and lingual surfaces in each category. This is probably because it is easier for the impression material to reach and register the details of occlusal and incisal areas than reaching the lingual and buccal areas, and this may be a

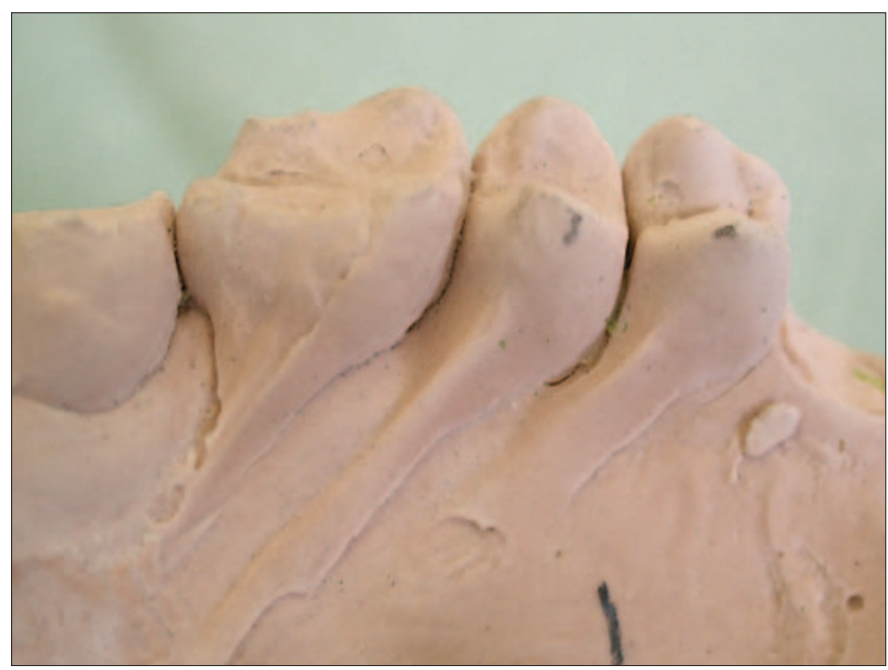

Fig. 4 Example of a dental cast illustrating inaccurate representation of the lingual surfaces of maxillary posterior teeth

reflection of the usual path of insertion of impression trays. An additional factor might also be that impression material could be displaced by the action of the tongue and cheeks before the tray is fully seated.

Poorer cast quality may also result from the difficulty sometimes experienced when seating the loaded impression tray in areas such as buccal anterior and the lingual and buccal areas of posterior regions in patients' mouths.

Given that working impressions are usually made with more accurate materials than those used for impressions of the opposing arch, it might be expected that working casts would generally have better quality than opposing casts. However, this was found not to be the case, as in the majority of instances the quality of the opposing casts was better than the working cast. This unexpected result might indicate that the difficulties encountered in attempting to capture the detail of the preparation, together with the remaining arch of teeth leads to a reduction in impression quality. This may be compounded by the use of materials of differing viscosity and manipulation characteristics in combination, as is usually the case when attempting to make a working impression. In addition the inherently hydrophobic nature of silicone impression materials may lead to difficulties if the tooth surfaces which the material is attempting to record are wet. This contrasts with a "simple' alginate impression for the opposing arch using a single viscosity material with which the clinician is more familiar, and also in comparison with silicone is relatively hydrophilic, and will therefore more easily record wet tooth surfaces. It was also noticed that 'drags' occurred in certain maxillary working casts starting from the distal parts of the palatal surfaces of posterior teeth and running postero-inferiorly (Fig. 4). This may be attributed to the clinical technique used in making the impression, particularly the path of insertion of the impression tray, driving the elastic impression material upwards and backwards at a later stage of the setting reaction.

\section{CONCLUSION}

The quality of opposing casts was significantly better $(\mathrm{P}<0.001)$ than that of the working casts. This unexpected result might indicate that some difficulty is experienced in handling the impression materials used in making the working impression (eg dual impression technique with elastic impression materials) compared with a 'simple' alginate impression of the opposing arch.

The quality of casts in the preparation areas was significantly better $(\mathrm{P}<0.001)$ than that of the areas remote from the preparation in the same arch. This result indicates that perhaps greater attention is being focused on the preparation to the detriment of the 


\section{RESEARCH}

quality of the impression in other areas. When using potentially hydrophobic materials, such as the silicones, the clinician should always dry the tooth surfaces of the unprepared teeth as well as any tooth preparation(s).

This study has demonstrated the need for continued vigilance on the part of the clinician in terms of quality control in relation to impression making and the dental casts obtained from them.

1. Winstanley R B. Crown and bridge impressions- a comparison between the UK and a number of other countries. Eur J Prosthodont Restor Dent 1999; 7: 61- 64.
2. Shillingburg H T, Hobo S, Whitsett L D, Jacobi R, Brackett S E. Fundamentals of fixed prosthodontics. 3rd ed. P 309. Chicago, IL: Quintessence Publishing Co, 1997.

3. British Society for Restorative Dentistry. Guidelines for crown and bridge. Eur J Prosthodont Restor Dent 1999: 7:3-9.

4. Carrotte PV, Winstanley R B, Green J R. A study of the quality of impressions for anterior crowns received at a commercial laboratory. Br Dent J 1993; 174: 235- 240.

5. Winstanley R B, Carrotte PV , Johnson A. The quality of impressions for crowns and bridges received at commercial dental laboratories. Br Dent J 1997: 183: 209- 213.

6. Randall R C, Wilson M A, Setcos J C, Wilson N H F. Impression materials and techniques for crown and bridgework: a survey of undergraduate teaching in the UK. Eur J Prosthodont Restor Dent 1998; 6: 75- 78

7. Davies S J, Gray R M, Smith PW. Good occlusal practice in simple restorative dentistry. BrDent J 2001; 191:365-368. 